\title{
Happy hypoxemia: A perplexing clinical entity in coronavirus disease 2019
}

\author{
Chithira V. Nair ${ }^{1}$, Dipu T. Sathyapalan ${ }^{2}$, Merlin Moni ${ }^{2}$, Akshaya Suresh $^{1}$, Pushpa Raghavan Roshni $^{1 *}$ \\ ${ }^{1}$ Department of Pharmacy Practice, Amrita School of Pharmacy, Amrita Vishwa Vidyapeetham, Kochi, India. \\ ${ }^{2}$ Department of Internal Medicine, Amrita School of Medicine, Amrita Vishwa Vidyapeetham, Kochi, India.
}

\begin{tabular}{l}
\hline ARTICLE INFO \\
\hline Received on: $25 / 02 / 2021$ \\
Accepted on: $24 / 09 / 2021$ \\
Available Online: $05 / 01 / 2022$
\end{tabular}

\section{Key words:}

COVID-19, dyspnea, happy hypoxemia, neural hypothesis, nucleus tractus solitaris, oxyhemoglobin dissociation curve.

\begin{abstract}
The novel pandemic, coronavirus disease 2019 (COVID-19), is a public health exigency of global concern with expanding cases worldwide. Severe acute respiratory syndrome-corona virus- 2 is the virus that accounts for COVID-19. The disease manifests with a wide array of symptoms ranging frommild upper respiratory tractinfection to severe pneumonia and death. The Happy hypoxemia, synonymously Silent hypoxemia, is described as a clinical entity in individuals with COVID-19, generally expressed as objective hypoxemia lacking respiratory distress symptoms. The condition is characterized by a drop in oxygen saturation and varying arterial blood gas. Intrapulmonary shunting, intravascular microthrombi, loss of lung perfusion control, and impaired diffusion capacity contributes to hypoxemic etiology in COVID-19. The ventilation-perfusion mismatch, covering from shunts to alveolar dead space ventilation, is the principal distinctive feature, suggesting numerous medical goals.
\end{abstract}

\section{INTRODUCTION}

The coronavirus disease 2019 (COVID-19) is a deadly infectious pandemic in late 2019 that intimidates human health and public safety. The coronaviruses are enfolded positive-sense RNA viruses with spike-like projections on their surface resembling a crown under the microscope. The virus is transmitted through large droplets produced during coughing and sneezing by the COVID-19 infected patients (Singhal, 2020). The clinical manifestations of COVID-19 range from symptomless state to acute respiratory distress syndrome (ARDS) and multiple organ dysfunction syndrome. The typical clinical manifestations cover headache, fever (not seen in all), cough, drowsiness, pharyngitis, myodynia, conjunctivitis, and dyspnea (Wang et al., 2020). One critical feature of COVID-19 that perplexes the clinician in coping with pneumonia is that some patients present with exceedingly low blood oxygenation but with no breathing difficulty. This occurrence has

\footnotetext{
*Corresponding Author

P. R. Roshni, Amrita School of Pharmacy, Amrita Vishwa Vidyapeetham, Kochi,India.E-mail: roshnipr@aims.amrita.edu
}

given rise to the term "Happy hypoxemia" (Velou and Ahila, 2020). Various reports disclosed that the occurrence of happy hypoxemia in coronavirus infected patients ranges from $20 \%$ to $40 \%$ (Rahman et al., 2021). A retrospective study conducted in France revealed that asymptomatic hypoxemia was associated with a very poor clinical outcome (33.3\% were shifted to intensive care unit (ICU) and $25.9 \%$ died) in COVID-19 patients (Brouqui et al., 2021).

\section{Dyspnea as a Sensation}

Dyspnea is the medical term for shortness of breath, expressed as "air hunger," prominent from tachypnea or hyperpnea. The mechanoreceptor present in the airway tract and chest wall contributes to dyspnea. The activation of vagal irritant receptors appears to strengthen dyspnea. The donation of metabolic rate in regulating the sense of shortness of breath in severe subjects continues to be unclear. The central and peripheral chemoreceptors are top-known contributing factors of the respiratory drive. Variations in the partial pressure of carbon dioxide happens to be the principal constituent, inducing shifts in $\mathrm{pH}$ at the degree of both the central and peripheral chemoreceptor (Vaporidi et al., 2020). Hypoxemia exhibits a restrained part in the sensation of panting accomplished by cardiopulmonary diseased patients on 
the opposite of hypercapnia that generates per se shortness of breath. Individuals with dyspnea are not hypoxemic, but those who have generally experienced a subtle refinement in manifestations after hypoxemia is managed with supplemental oxygen therapy (Harikrishnan et al., 2018). Dyspnea often experiences when the arterial partial pressure of oxygen $\left(\mathrm{PaO}_{2}\right)$ falls below $40 \mathrm{mmHg}$. Hyperventilation gives rise to reduced partial Pressure of carbon dioxide $\left(\mathrm{PaCO}_{2}\right)$, causing constriction of arterioles, consequently reducing cerebral blood flow, and intracranial pressure (ICP). On the contrary, the increase of $\mathrm{PaCO}_{2}$ promotes expanded ICP, eventually declining the degree of consciousness, impaired brain stem reflexes, and impaired postural and motor feedback. Proper awareness concerning the pathophysiological determinants of hypoxemia may help develop a more concluded understanding of a corona patient's therapeutic manifestations as well as treatment (Richards, 2017).

\section{Happy/Silent Hypoxemia}

Happy hypoxemia can be quoted as one of the most distinctive and interesting features so far observed in any disease. The patients seem to be normal, but the problem with this condition is that its oxygen saturation $\left(\mathrm{SpO}_{2}\right)$ seems very less when compared to its normal levels (95\% or more) (Velou and Ahila, 2020). When the $\mathrm{SpO}_{2}$ falls to $90 \%$ or less, as seen in pneumonia affected patients experience dyspnea, drowsiness, etc. The reason is that the capacity of the lungs to inflate is affected. As a result, the proper exchange of gas is not possible. Hence the carbon dioxide level gets increased (Viswanathan et al., 2018). In COVID-19, not only the lung is affected but more so the perfusion. The swelling or inflammation of capillaries results in clot formation due to which tissues do not get fully oxygenated. But since lungs dilate and constrict fairly and almost like normal, no signs and symptoms of hypoxemia are exhibited by the patients and they don't seem to be in any kind of distress (Chandra et al., 2020). The pulse oximetry used to measure $\mathrm{SpO}_{2}$ is usually used to detect hypoxemia (Guo et al., 2017). Happy hypoxemia is not only present in corona patients but in individuals having atelectasis, arterio-venous malformations, or right-to-left intracardiac shunt. In the starting stage of COVID-19, numerous principles come up with the evolution of arterial hypoxemia, without an accompanying rise in the work of respiration. Expeditious decadence may happen. A rigorous recognition of the pathophysiological factors of hypoxemia may assist in further understanding of a patient's symptoms and treatment (Anoop and Verma, 2020).

\section{Difference Between Hypoxemia and Happy Hypoxemia}

Hypoxemia is a medical condition that happens due to a low concentration of oxygen in the blood. The arterial oxygen level around 75-100 $\mathrm{mmHg}$ is considered normal, and if declined below $90 \%$, the patient may experience hypoxemia. The difference between the characteristics of normal hypoxemia and happy hypoxemia is shown in Table 1 (Haryalchi et al., 2021).

\section{Variations in Oxygen-Hemoglobin Dissociation Curve}

Hypoxemia is detected by pulse oximetry. The $\mathrm{SpO}_{2}$ needs to be elucidated with care in severe acute respiratory syndrome-corona virus-2 (SARS-CoV-2). The oxygen-hemoglobin dissociation curve with sigmoid shape appears to shift to the
Table 1. Difference between hypoxemia and happy hypoxemia.

\begin{tabular}{cc}
\hline Hypoxemia & Happy hypoxemia \\
\hline $\begin{array}{c}\mathrm{PaO}_{2} \text { in the blood decreases results } \\
\text { in dyspnea. }\end{array}$ & $\begin{array}{c}\mathrm{PaO}_{2} \text { in the blood decreases, but the } \\
\text { patient appears to be normal without } \\
\text { dyspnea. }\end{array}$ \\
Potential causes: & $\begin{array}{c}\text { Potential causes: } \\
\mathrm{CO}_{2} \text { level will be poor }\end{array}$ \\
Poor oxygenation & $\mathrm{CO}_{2}$ level will be normal \\
Status of breathing: poor & Poor oxygenation \\
Clinical manifestations: & Status of breathing: normal \\
Presence of dyspnea, tachypnea, & Clinical manifestations: \\
and tachycardia & Absence of dyspnea, tachypnea, and \\
\end{tabular}

left, as a result of persuaded respiratory alkalosis for the reason of hypoxemia derived fast and deep breathing (tachypneahyperpnea). Throughout the period of hypocapnia, the harmony of hemoglobin for oxygen and consequently $\mathrm{SpO}_{2}$ arises for a particular level of $\mathrm{PaO}_{2}$, describing why $\mathrm{SpO}_{2}$ is possibly well conserved in the face of an extremely poor $\mathrm{PaO}_{2}$. In high altitude hypoxemia, this uncovering is seen, in which hypocarbia shifts the oxyhemoglobin dissociation curve and enhance blood $\mathrm{SpO}_{2}$. The alveolar gas equation also foretells that hyperpnea and the resulting fall in the alveolar $\mathrm{PaCO}_{2}$ lead to a rise in the alveolar $\mathrm{PaO}_{2}$ and eventually result in the rise of $\mathrm{SpO}_{2}$ (Tobin et al., 2020). There is a biological hypothesis describing the leftward shift of the curve in SARS-CoV-2. Concerning the hypothesis, serum levels of heme are raising in COVID-19 accompanying deleterious irons ions, developing an infection, and cell death. This results in the formation of a huge amount of serum ferritin to attach these free irons to lessen the destruction of tissue (Dhont et al., 2020).

\section{ETIOLOGY OF HYPOXEMIA IN COVID-19}

\section{Intrapulmonary shunting}

A low oxygen concentration in arterial blood present initially in COVID-19 is firstly due to ventilation-perfusion mismatch and hence perseverance of pulmonary arterial blood flow to non-ventilated alveoli, ruminated by a noticeable rise in $\mathrm{P}(\mathrm{A}-\mathrm{a}) \mathrm{O}_{2}$ gradient. The infection results in moderate local interstitial edema. As a result of increased edema, deprivation of surfactant, and superimposed pressure, alveolar collapse arises and a considerable portion of the cardiac output is perfusing nonaerated pulmonary tissue, leads to (Fig. 1) intrapulmonary shunting (Gattinoni et al., 2020). The increase in tidal air throughout the disease results in increasing negative inspiratory intrathoracic pressure. The increase in pulmonary permeability by cause of inflammation leads to continuous edema, alveolar flooding, and patient self-inflicted lung injury. The increased edema will later increase the weight of the lung, alveolar collapse, and dependent atelectasis leading to enhanced shunt fraction and promote a decrease of oxygenation which may not fully be rectified by improving fraction of inspired oxygen (Dhont et al., 2020).

\section{Loss of lung perfusion regulation}

During COVID-19 infection the failure of hypoxic pulmonary vasoconstriction results in the appearance of a persistent increase in pulmonic blood flow to unaired lung 


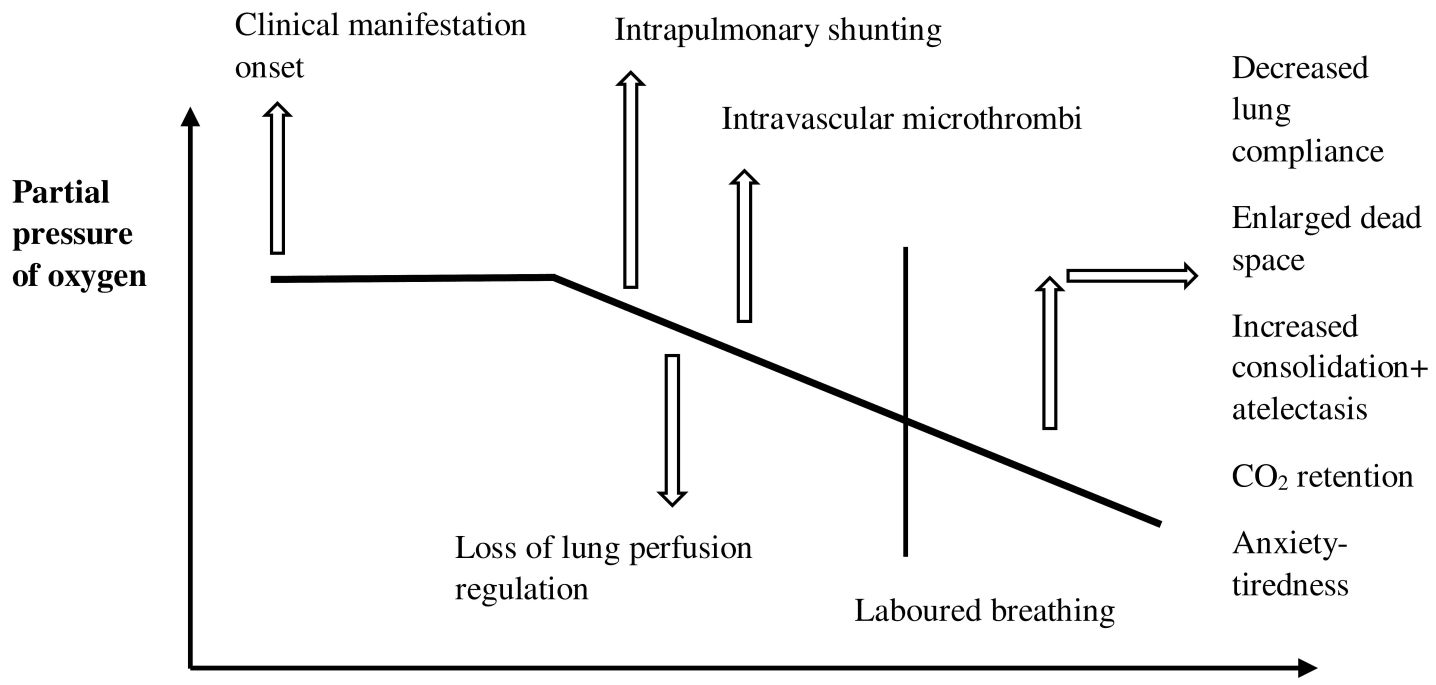

Time course

Figure 1. Causes of hypoxemia in COVID-19.

alveoli. Vasoplegia also seems related to the loss of lung perfusion regulation (Fig. 1). Additionally, the imperfect regulation of the renin-angiotensin system (RAS) donates to the etiology of COVID-19. Angiotensin-converting enzyme (ACE2) is the cell receptor of COVID-19 for entering a cell. The enzyme converts angiotensin 11 to angiotensin 1-7, furthermore responsible for bradykinin degradation. The decrease in ACE2 results in elevated Ang 11, activating pulmonary vasoconstriction (Tay et al., 2020).

\section{Intravascular microthrombi}

A major hallmark of the pathophysiology of COVID-19 is endothelial injury. The cytopathic virus can damage lung capillary endothelial cells that indicate ACE2. A variation between procoagulant and fibrinolytic activity in the existence of acute inflammation and endothelial damage results in intravascular microthrombi (Fig. 1). The pro-coagulant activity may be due to complement system-mediated activation of coagulation or may happen because of blocking of plasminogen activation and fibrinolysis. The endothelial deliverance of tissue factor and triggering of clotting factor $\mathrm{V} 11$ and $\mathrm{X} 1$ results in diffuse intravascular coagulation in severe corona patients (Zhang et al., 2020). A large number of COVID-19 patients exhibit an increased measure of D-dimer resulting in the formation of blood clots. The uncontrolled clot formation results in ventilation/perfusion (V/Q) mismatch (a condition in which one or more part of the lung receives oxygen without blood flow or blood flow without oxygen) and damage to pulmonary tissue. Furthermore, clotting is also regulated by stimulating C-reactive protein and developing complement stimulation and hepatic fibrinogen production as an acute phase protein COVID-19 (Tian et al., 2020).

\section{Altered diffusion capacity}

There is a direct relationship between altered diffusion capacity and the severity of the disease. In COVID-19 patients the pulmonary diffusion capacity may be altered (Fig. 1), however, pure diffusion problems are occasionally a reason for enhanced
$\mathrm{P}(\mathrm{A}-\mathrm{a}) \mathrm{O}_{2}$ (alveolar-arterial oxygen gradient) at rest (Dhont et al., 2020). The basement membrane is enfolded with debris, comprised of Factor 1a, dead cell, and complement activation product due to deprivation of alveolar epithelial cells and a pro-coagulant state. The hyperdynamic pulmonic circulation shall not provide proper time for $\mathrm{RBC}$ to equilibrate their oxygen absorption with incremental physical activities (exercise) and in the situation of absent hypoxic constriction of blood vessels in COVID-19. As a result, alteration occurs in diffusion leading to elevated $\mathrm{P}(\mathrm{A}-\mathrm{a}) \mathrm{O}_{2}$ gradient and exercise prompted arterial hypoxemia (Ziehr et al., 2020).

\section{Neural Hypothesis of Happy Hypoxemia}

The nucleus tractus solitaris (NTS) is the principal visceral sensory nucleus in the brain. It is situated in the dorsomedial medulla oblongata. It collects baroreceptors and chemoreceptors afferents from the glossopharyngeal nerve. It receives visceral afferents from most of the organs via the vagus nerve by which it plays a major part in cardiovascular function, ventilation, gastro intestinal mobility, and immune-modulatory activities (Omer et al., 2020).

The coronavirus infects respiratory tract (upper and lower). They get into the body via the nasal or oral cavity. After reaching the oral cavity and pharynx, the virus may propagate together with the axons of cranial nerves $\mathrm{V}, \mathrm{V} 11,1 \mathrm{X}$, and $\mathrm{X}$. These cranial nerves relay the NTS result in inflammation. The carotid body consists of a chemoreceptor that is initially operated by a depletion in the arterial $\mathrm{PaO}_{2}$. Low oxygen in the blood stimulates the carotid body chemoreceptor, and the afferent signals are transferred at the NTS via the glossopharyngeal nerve. This results in an increased respiratory rate and vasoconstriction. In COVID-19 arbitrated infection of NTS, the afferent hypoxia stimuli from carotid bodies may not be successfully relayed at the NTS, followed by an inappropriate efferent lung response. This is likely considered as the rationale for normal breathing in the presence of severe hypoxemia during COVID-19 (GregoryCutsforth and Benarroch, 2017). 


\section{Happy Hypoxemia to ARDS}

The most distinctive and demanding symptoms of COVID-19 are connected to ARDS. An approximate of $46 \%-65 \%$ of patients in ICU worsen in a short period and died because of respiratory failure. Reduced perception of dyspnea slows down patients from looking for medical care finally results in death. Medical groups must recognize the happy hypoxemia in COVID-19, which helps the doctors to provide proper treatments thereby reduce the risk of complications and death (Anoop and Verma, 2020).

\section{Management Strategies}

The major therapeutic strategies include giving antiviral agents, management of $\mathrm{V} / \mathrm{Q}$ mismatch, and cytokine storm reduction. Considering the circulation of blood through tissues, circumventing clump of fibrin, platelets, and red blood cells, and continuing deposition of fibrinoid material is one of the corrective policies (Tarry and Powell, 2017). The COVID-19 patients with D-dimer level high on admittance are beneficial for taking thromboprophylaxis. Currently, it is safe to use vascular activators for managing ARDS in COVID-19. Many trials are verifying the use of anti-inflammatory agents to prevent macro-and microthrombi. Euler-Liljestrand mechanism can be considered to enhance the matching of regional perfusion and airing in the alveolus (Slessarev et al., 2020). The RAS modulation may have an effective part in reinstating pulmonary perfusion management and nowadays many studies are in progress. In the case of ventilation, supplemental oxygen is the primary factor in providing oxygen. Many studies stated that awake proning position enhances oxygenation in corona disease (Leeladharan et al., 2018).

At this time, the underlying pathology the separation of happy hypoxemia and subtle dyspnea in COVID-19 remains unknown. In our experience, people with severe glossopharyngeal or vagus nerves lesions because of cranial nerve injury following neck cancer or associated neuropathies have this disassociation, though these observations are missing in the autopsy records unexpectedly and now appearing in COVID-19 cases. In the one hand, neurological manifestations such as headache, anosmia, impaired mental state, delirium, and epilepsy are normal in extreme COVID-19 cases; and cerebral spinal fluid with SARS-COV-2 and is believed to reach the brain via synapse-associated routes (Archer et al., 2020). Harm to afferent hypoxemia-sensing neurons in COVID-19 patients could be caused by an acute cytokine rush or through the action of SARS-COV2 on mitochondria or neuronal fibers directly (Wei-jie et al., 2020). On the other hand, results from the magnetic resonance imaging tests and brain pathology records of mortal COVID-19 cases, are inconclusive and may not have a pathological correlation to verify the absence of dyspnea (Robinson and Gebhart, 2008). The most frequent brain pathology results in fatal COVID-19 cases shows evident areas of ischemic and hemorrhagic strokes with only minimum inflammation (Burki and Lee, 2010). The neurological symptoms of other coronaviruses are less studied, although myopathy and neuropathy have been identified much in both SARS-CoV and other coronavirus strains (Coolen et al., 2020). The fact that interests me the most with COVID-19 is that this stage is the patient does not perceive and the brain does not reveal in terms of pathology.
Furthermore, of the pathology underlying, diminished experience of dyspnea is a condition due to the exchange of blood and gas. It can conceal the seriousness of a patient's medical condition, effectively delaying patients' access to urgent medical treatment. Patients admitted with COVID-19 will die suddenly after taking voluntary "cut-off" from oxygen regurgitation. "Happy hypoxemia" after recognizing as a feature of COVID-19 pneumonia has resulted in improved patient care, with doctors depending on other disease signs including tachycardia, fever, or serum inflammatory acute reactants to direct treatment or discharge. Continued studies into how the novel coronavirus affects peripheral sensors and neuronal circuits could lead to a better understanding of the virus's mechanisms (Chaturbhuj et al., 2021).

\section{CONCLUSION}

This global pandemic is changing the way we used to live. The physicians should not only believe in the patients behaving normally but proper evaluation of the number of breaths taken per minute by the patients, an indication of hyperpnea, $\mathrm{SPO}_{2}$, and intrusive measurement of decreased level of oxygen and carbon dioxide in the blood at proper intervals are required. Pulse oximetry needs to be elucidated by care, due to the left-sided shifting of the oxygen-hemoglobin dissociation curve. The happy hypoxemia is caused by intrapulmonary shunting, loss of lung perfusion regulation, intravascular microthrombi formation and altered diffusion capacity. As in the initials days of this condition, the respiratory system does not sense any disturbance in breathing. Later, quick respiratory decompensation happens, and tachypnea and hyperpnea experience. In the current COVID-19 situation the true outcomes of happy hypoxemia endured controversy, resulting in indistinct recommendations on how to handle such cases.

\section{AUTHOR CONTRIBUTIONS}

All authors made substantial contributions to conception and design, acquisition of data, or analysis and interpretation of data; took part in drafting the article or revising it critically for important intellectual content; agreed to submit to the current journal; gave final approval of the version to be published; and agree to be accountable for all aspects of the work. All the authors are eligible to be an author as per the international committee of medical journal editors (ICMJE) requirements/guidelines.

\section{FUNDING}

There is no funding to report.

\section{CONFLICTS OF INTEREST}

The authors report no financial or any other conflicts of interest in this work.

\section{ETHICAL APPROVALS}

This study does not involve experiments on animals or human subjects.

\section{PUBLISHER'S NOTE}

This journal remains neutral with regard to jurisdictional claims in published institutional affiliation. 


\section{REFERENCES}

Archer SL, Sharp WW, Weir EK. Differentiating COVID-19 pneumonia from acute respiratory distress syndrome (ARDS) and high altitude pulmonary edema (HAPE): therapeutic implications. Circulation, 2020; 142(2):101-4.

Brouqui P, Amrane S, Million M, Cortaredona S, Parola P, Lagier J, Raoult D. Asymptomatic hypoxia in COVID-19 is associated with poor outcome. Int J Infect Dis, 2021; 102:233-8.

Burki N, Lee L. Mechanisms of dyspnea. Chest, 2010; 138(5):1196-201.

Chandra A, Chakraborty U, Pal J, Karmakar P. Silent hypoxia: a frequently overlooked clinical entity in patients with COVID-19. BMJ Case Rep, 2020; 13(9):207-37.

Chaturbhuj R, Neeraj B, Ram BA, Shaik AJ, Siby Gopinath, Sujit S, Sandeep P, Turaga S, Sita J, Sangeeta R, Dinesh SN, Sanjay P, Kaushik R, Shyam KJ, Fayaz RK, Jagarlapudi MM, Kurupath R. Impact of COVID-19 pandemic on epilepsy practice in India: a tripartite survey. Seizure, 2021; 86:60-7.

Coolen T, Lolli V, Sadeghi N, Rovai A, Trotta N, Taccone fs, Creteur J, Henrad S, Goffard J, Dewitte O, Naeije G, Goldman S, Tiege $\mathrm{X}$. Early postmortem brain MRI findings in COVID-19 non-survivors. Neurology, 2020; 95(14):101-16.

Dhont S, Derom E, Braeckel EV, Depuydt P, Lambrecht BN. The pathophysiology of "happy" hypoxemia in COVID-19. Respir Res, 2020; 21(1):198.

Gattinoni L, Chiumello D, Caironi P, Busana M, Romitti F, Brazzi L, Camporota L. COVID-19 pneumonia: different respiratory treatment for differentphenotypes? Intensive Care Med, 2020; 46(6):1099-02.

Gregory-Cutsforth JK, Benarroch EE. Nucleus of the solitary tract, medullary reflexes, and clinical implications. Neurology, 2017; 88(12):1187-96.

Guo L, Bobhate P, Kumar S, Vadlamudi K, Kaddoura T, Elgendi M, Holinski P, Coe JY, Rutledge J, Adatia L. Hyperoxia reduces oxygen consumption in children with pulmonary hypertension. Pediatr Cardiol, 2017; 38(5):959-64.

Harikrishnan S, Sanjay G, Ashishkumar M, Menon J, Rajesh GN, Kumar RK, Koshy AG, Attacheril TV, George R, Punnoose E, Arshraf SM, Arun SR, Cholakkal M, Jeemon P. Pulmonary hypertension registry of Kerala, India (PRO-KERALA) - clinical characteristics and practice patterns. Int I Cardiol, 2018; 265:212-7.

Haryalchi K, Heirdarzadeh A, Abedinzaide M, Olangian-Tehrani S, Tehran S. The importance of happy hypoxemia in COVID-19. Anesth Pain Med, 2021; 11(1):1118-22.

Leeladharan SP, Jayashankar JP, Kottayil BP, Kappanayil M, Raman K, Balachandran R. pulmonary hemorrhage due to unrecognized bronchial collateral after arterial switch operation. Ann Thorac Surg, 2018; 105(3):117-8.

Omer SB, Malani P, Rio CD. The COVID-19 pandemic in the US: a clinical update. JAMA, 2020; 323(18):1767-8.

Rahman A, Tabassum T, Araf Y, Nahid AA, Ullah A, Hosen MJ. Silent hypoxia in COVID-19: pathomechanism and possible management strategy. Mol Biol Rep, 2021; 48:3863-9.
Richards JB. Calculated decisions: mMRC (modified Medical Research Council) dyspnea scale. Emerg Med Pract, 2017; 19(10):1-2.

Robinson D, Gebhart F. Inside information-the unique features of visceral sensation. Mol Intery, 2008; 8(5):242-53.

Singhal T. A review of coronavirus disease-2019 (COVID-19). Indian J. Pediatr, 2020; 87(4):281-6.

Tarry D, Powell M. Hypoxic pulmonary vasoconstriction. BJA Educ, 2017; 17(6):208-13.

Slessarev M, Cheng J, Ondrejicka M, Arntfield R. Patient selfproning with high-flow nasal cannula improves oxygenation in COVID-19 pneumonia. Can J Anesth, 2020; 67(9):1288-90.

Tay MZ, Poh CM, Renia L, MacAry PA, Ng LFP. The trinity of COVID-19: immunity, inflammation and intervention. Nat Rev Immunol Nat Res, 2020; 20(6):363-74.

Tian S, Hu W, Niu L, Liu H, Xu H, Xiao S-Y. Pulmonary pathology of early-phase 2019 novel coronavirus (COVID-19) pneumonia in two patients with lung cancer. J Thorac Oncol, 2020; 15(5):700-4.

Tobin MJ, Laghi F, Jubran A. Why COVID-19 silent hypoxemia is baffling TP physicians. Am J Respir Crit Care Med, 2020; 202(3):356-60.

Anoop UR, Verma K. Happy hypoxemia in COVID-19. ACS Chem. Neurosci, 2020; 11(13):1865-7.

Vaporidi K, Akoumianaki E, Telias I, Goligher EC, Brochard L, Georgopoulos D. Respiratory drive in critically ill patients pathophysiology and clinical implications. Am J Respir Crit Care Med, 2020; 201(1):20-32.

Velou MS, Ahila E. Happy hypoxemia: what has been forgotten. Int Arch Integr Med, 2020; 7(8):75-9.

Viswanathan K, Rakesh PS, Balakrishnan S, Shanavas A, Dharman V. Prevalence of chronic respiratory diseases from a rural area in Kerala, southern India. Indian J Tuberc, 2018; 65(1):48-51.

Wang C, Horby PW, Hayden FG, Gao GF. A novel coronavirus outbreak of global health concern. Lancet, 2020; 395(10223):470-3.

Wei-jie G, Zheng-yi N, Yu H, Wen-hua L. Clinical characteristics of coronavirus disease 2019 in China. N Engl J Med, 2020; 382:1708-20.

Zhang L, Yan X, Fan Q, Liu H, Liu X, Liu Z, Zhang Z. D-dimer levels on admission to predict in-hospital mortality in patients with Covid-19. J Thromb Haemos, 2020; 18(6):1324-9.

Ziehr DR, Alladina J, Petri CR, Maley JH, Moskowitz A, Medoff BD, Hibbert KA, Thompson BT, Hardin CC. Respiratory pathophysiology of mechanically ventilated patients with COVID-19: a cohort study. Am J Respir Crit Care Med, 2020; 201(12):1560-4.

How to cite this article:

Nair CV, Sathyapalan DT, Moni M, Suresh A, Roshni PR. Happy hypoxemia: A perplexing clinical entity in coronavirus disease 2019. J Appl Pharm Sci, 2022; 12(01):065-069. 